\title{
Protein folding/refolding analysis by mass spectrometry
}

\author{
Scrambling of disulphide bridges in insulin
}

\author{
Howard R. MORRIS, ${ }^{*} \S$ Piero PUCCI, $\dagger$ Maria PANICO* and Gennaro MARINO $\ddagger$ \\ *Department of Biochemistry, Imperial College of Science, Technology and Medicine (University of London), Exhibition Road, \\ London SW7 2AY, U.K., †Istituto Chimico, Università della Basilicata, 85100 Potenza, Italy, and †Dipartimento di Chimica \\ Organica e Biologica, Università di Napoli and Servizio di Spettrometria di Massa, Consiglio Nazionale delle Ricerche-Università \\ di Napoli, 80134 Napoli, Italy
}

\begin{abstract}
In this paper we present a protocol that allows a dynamic analysis of disulphide-bridge formation, based on freezing the intermediates by acid/acetone precipitation, followed by digestion with pepsin and direct fast-atom-bombardment massspectrometric analysis. A rapid definition of the exact nature of disulphide bridges formed can be obtained via a definitive assignment of disulphide-linked peptides according to their unique mass values. With the use of an appropriate thiol concentration, scrambling of the native disulphide bonds in bovine insulin occurs, and the process is catalysed by protein disulphide-isomerase (EC 5.3.4.1). The disruption of native and the formation of new disulphide bonds can be monitored as described above, and interestingly B-chain dimers containing Cys-B7-Cys-B7 and Cys-B7-Cys-B19 bonds are detected.
\end{abstract}

\section{INTRODUCTION}

The analysis of protein folding/refolding is a problem of considerable interest in the field of protein chemistry, both in theoretical studies [1] and in practical applications, for example expression of recombinant proteins [2].

The folding pathway of proteins possessing native disulphide bridges can be determined experimentally, since the disulphide bonds present at any one time can be trapped by irreversibly blocking all free thiol groups and thereafter keeping the trapped species under conditions where their disulphide bridges are stable. Such an approach has been extensively exploited by Creighton [3], who trapped the intermediates by alkylation with iodoacetic acid, separated them by ion-exchange chromatography and finally located bridged peptides by the diagonal electrophoretic method. In this way the disulphidebridging/folding pathways have been determined to various extents for bovine pancreatic trypsin inhibitor [4] and for ribonuclease A [5]. Recently, however, these results have been questioned [6,7], and the need for an independent method of analysis is apparent.

We have previously introduced a new technique for the analysis of disulphide bridges in proteins, and suggested that the method would be applicable to dynamic analysis of the protein folding, the results of which are reported in the present paper. This method is based upon determination of the masses of disulphidelinked peptides by the direct analysis of unfractionated or partially purified protein digests by high-field fast-atombombardment mass spectrometry (f.a.b.-m.s.) [8].

The results described in the present paper demonstrate that this m.s. method allows sampling of the folding/refolding experiment on a time-course basis, and, in conjunction with reduction and Edman degradation, gives rapid definition of the exact nature of the disulphide bridges formed at any particular time, via a definitive assignment of disulphide-linked peptides according to their unique mass values.

\section{MATERIALS AND METHODS}

Bovine insulin, dithiothreitol (DTT), pepsin and glycerol were purchased from Sigma Chemical Co. (St. Louis, MO, U.S.A.). All other reagents were of analytical grade. Bovine insulin $(4 \mathrm{mg})$ was dissolved in $750 \mu \mathrm{l}$ of $50 \mathrm{~mm}$-sodium phosphate buffer, $\mathrm{pH} 7.5$, containing $1 \mathrm{~mm}$-EDTA and treated with $20 \mu \mathrm{l}$ of DTT solution of various concentrations to give either full or partial reduction of the disulphide bonds. Incubation was carried out at room temperature for up to $4 \mathrm{~h}$. Insulin was precipitated by adding $10 \mathrm{ml}$ of acid/acetone $(1 \mathrm{M}-\mathrm{HCl} /$ acetone, $1: 39, \mathrm{v} / \mathrm{v})$; the precipitate was collected by centrifugation at $2000 \mathrm{~g}$ for $15 \mathrm{~min}$, washed and dried.

A final DTT concentration of $3 \mathrm{~mm}$ was found to give full reduction of the two interchain disulphide bridges. Scrambling experiments were carried out by incubating the same amount of insulin with a $1 \mathrm{mM}$ final concentration of DTT in $1 \mathrm{ml}$ of sodium phosphate buffer as described above; a precipitate developed, visible from $30 \mathrm{~min}$. Time-course samples were withdrawn after $1,2,3$ and $4 \mathrm{~h}$ and precipitated with acid/acetone. In some experiments the time-course samples were centrifuged to remove the precipitate formed following DTT addition and the supernatant was treated with acid/acetone.

Disulphide scrambling in the presence of the enzyme protein disulphide-isomerase (PDI, EC 5.3.4.1; kindly given by Dr. R. Freedman, University of Kent, Canterbury, Kent, U.K.) was carried out by adding $40 \mu \mathrm{l}$ of a $1 \mathrm{mg} / \mathrm{ml}$ PDI solution to the incubation mixture. The precipitate developed in 5-10 $\mathrm{min}$; timecourse samples were taken from $15 \mathrm{~min}$ to $2 \mathrm{~h}$.

Peptic digestion of the samples was performed in $5 \%(\mathrm{v} / \mathrm{v})$ formic acid at $37^{\circ} \mathrm{C}$ for $6 \mathrm{~h}$ at an enzyme/substrate weight ratio of $1: 50$.

M.s. analysis of the samples was carried out on a high-field ZAB HF instrument (VG Analytical, Manchester, U.K.) fitted with an M-Scan (Ascot, Berks., U.K.) f.a.b. gun and ion source, the gun producing $10 \mathrm{keV}$ xenon ions as bombarding species.

Abbreviations used: f.a.b.-m.s., fast-atom-bombardment mass spectrometry; DTT, dithiothreitol; PDI, protein disulphide-isomerase.

$\S$ To whom correspondence should be addressed. 


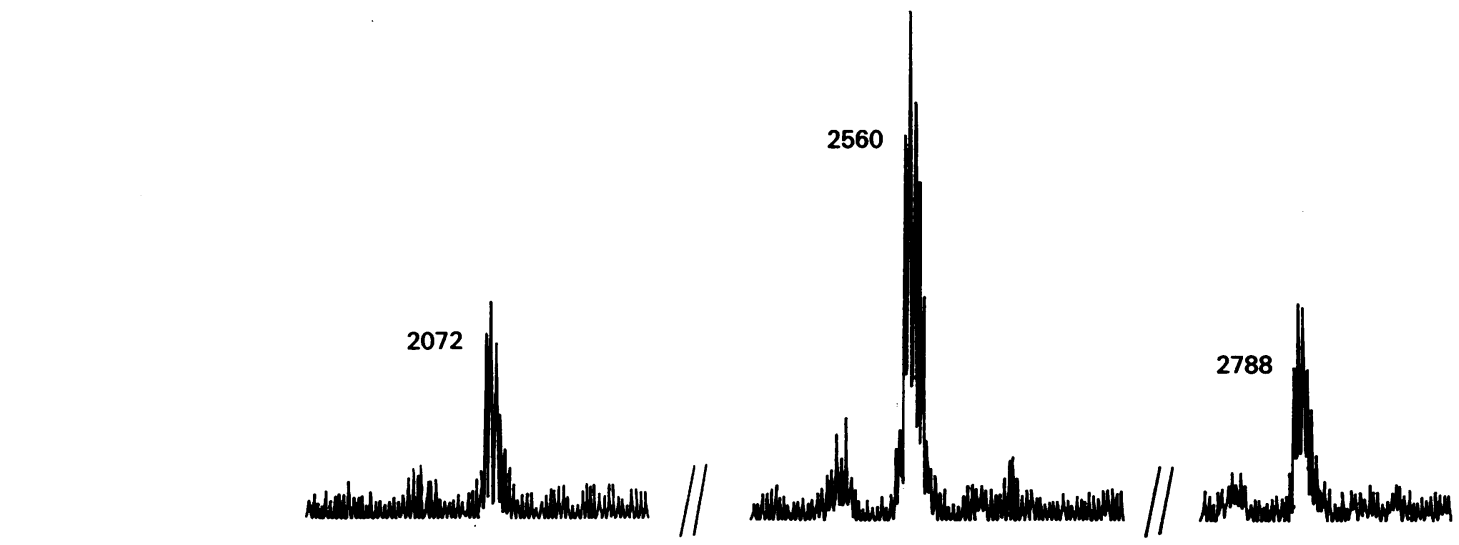

Fig. 1. Partial f.a.b.-m.s. map showing the high-mass region of the native non-reduced insulin following peptic digestion

The ${ }^{12} \mathrm{C}$-containing components of the isotope clusters are labelled with their nominal mass, and these values are used to compute the peptide composition of each. For interpretation see the text.

Table 1. Observed mass values in the peptic digest of reduced insulin

The peptide sequence corresponding to each quasimolecular ion $\left(M \mathrm{H}^{+}\right)$is given in the right-hand column (see the text).

\begin{tabular}{rc}
\hline $\mathbf{H}^{+}$ & Peptide \\
\hline 408 & B15-17 \\
417 & A1-4 \\
431 & B12-15 \\
497 & B16-19 \\
508 & B26-29 \\
526 & B22-25 \\
579 & B26-30 \\
683 & B16-21 \\
712 & B20-25 \\
726 & B25-30 \\
766 & B5-11 \\
880 & B17-24 \\
914 & B18-25 \\
994 & B5-13 \\
1027 & B16-24 \\
1043 & B17-25 \\
1190 & B16-25 \\
1254 & B1-11 \\
1309 & A1-13 \\
1482 & B1-13
\end{tabular}

Samples were dissolved in $5 \%(\mathrm{v} / \mathrm{v})$ acetic acid and loaded on to a glycerol-coated probe tip. Approx. 1-10 nmol of sample was used to generate data. In addition to considerations of the mass observed and the specificity of the enzyme used, assignments of signals in the mass spectra are concluded by using Edman degradation and reduction methods previously described for insulin [8].

\section{RESULTS}

Fig. 1 shows the partial mass spectrum (high-mass region) of the native non-reduced insulin, following peptic digestion. The interpretation of these and similar data has been reported previously [8]. Signals at $m / z 2072,2560$ and 2788 are assigned to the disulphide-bridged peptides A1-13+B5-11, A1-13+B1-11 and $A 1-13+B 1-13$ respectively. In the native molecule these species carry an intrachain disulphide bridge between Cys-A6 and Cys-A11 and interchain disulphide bridge between Cys-A7 and Cys-B7.

In the research reported here, the first objective was to map a reduced preparation of insulin. At a concentration of DTT giving an approximately 2-fold excess of thiol over disulphide bridges, the f.a.b.-m.s. map showed the signals listed in Table 1, assignable to individual peptic peptides, indicating full reduction of the two interchain disulphide bonds. It is worth noting that the reduction of the interchain disulphide bond Cys-A6-CysA11 is not observed in the above conditions, as demonstrated by the presence of a peak at $m / z 1309$ occurring 2 mass units lower than the calculated mass value for the fully reduced $\mathrm{Al}-13$ peptide. This is in agreement with previous reports that the disulphide bond Cys-A6-Cys-A11 is not reduced in nondenaturing conditions, being buried in the interior of the molecule (for a review see ref. [9]).

The signals in Table 1, together with the disulphide-bridge signals of the native molecule, can now be used as a template or reference position from which to monitor the oxidative pathway towards re-formation of disulphide bridges, whether natural or otherwise.

From this starting point, the next objective was to test the ability of this new methodology to monitor the re-formation of disulphides by using a precise physicochemical method allowing definition of the actual disulphide bridges formed. Two approaches were used in this study: firstly incubation of insulin with a low concentration of reducing agent, and secondly catalysis by PDI.

When insulin was incubated with a low concentration of reducing agent, giving no excess of thiol over disulphide bridges, the clear solution became cloudy within $30 \mathrm{~min}$. In time-course experiments samples of the mixture were precipitated with acid/acetone as described in the Materials and methods section. As an example, Fig. 2 shows the f.a.b.-m.s. map of the peptic digest of the acid/acetone precipitate at the $2 \mathrm{~h}$ incubation point. It is interesting to note that the spectrum can be interpreted as a combination of 'native' molecules carrying the disulphide bonds observed in Fig. $1(\mathrm{~m} / z$ 2072, 2560 and 2788) plus scrambled molecules derived from B-chains via new signals at $m / z 2278,2441,2506,2554,2669,2733,2804$ and 2961. The assignment of these new signals is reported in Table 2 .

Confirming this diagnosis of disulphide-bridge re-arrangement or scrambling, the removal of the incubation precipitate from the time-course samples by centrifugation, and the addition of acid/acetone to the supernatant, led to the separation of 

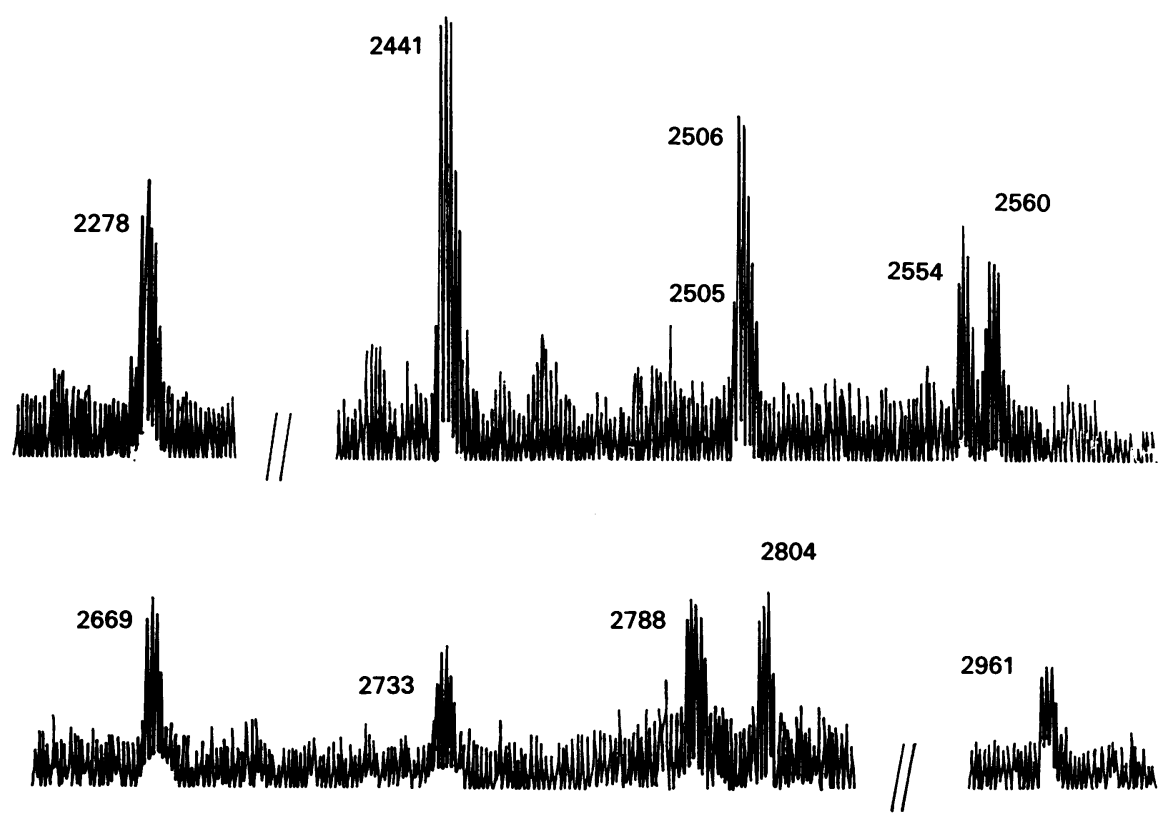

Fig. 2. Partial f.a.b.-m.s. map showing the high-mass region of a peptic digest of the total sample from an insulin-scrambling experiment taken at the $2 \mathrm{~h}$ incubation point

The spectrum contains signals derived from native and scrambled disulphide bridges. For interpretation see the text and Table 2.

Table 2. Mass values of disulphide-bridged peptides from a peptic digest of scrambled insulin

\begin{tabular}{lc}
\hline$M \mathrm{H}^{+}$ & Peptides \\
\hline 2278 & B1-11+B17-25 \\
2441 & $\mathrm{~B} 1-11+\mathrm{B} 16-25$ \\
2506 & $\mathrm{~B} 1-13+\mathrm{B} 17-25$ \\
2554 & $\mathrm{~B} 1-11+\mathrm{B} 15-25$ \\
2669 & $\mathrm{~B} 1-13+\mathrm{B} 16-25$ \\
2733 & $\mathrm{~B} 1-13+\mathrm{B} 1-11$ \\
2804 & $\mathrm{~B} 1-11+\mathrm{B} 1-14$ \\
2961 & $\mathrm{~B} 1-13+\mathrm{B} 1-13$
\end{tabular}

'scrambled' and 'native' insulin. Fig. 3 shows the f.a.b.-m.s. map of the $2 \mathrm{~h}$-incubation precipitate showing only B-chain-derived signals defining the existence of two new disulphide bridges formed between Cys-B7 and Cys-B19 and between Cys-B7 and Cys-B7. The analysis of the corresponding acid/acetone pre- cipitate showed the occurrence of disulphide-bridge-containing peptides derived only from the native insulin. After the removal of the incubation precipitate at the $4 \mathrm{~h}$ point there was no further precipitate on the addition of acid/acetone to the supernatant, indicating that full scrambling had occurred within this time.

Experiments using PDI gave very similar results but with a substantial increase in scrambling rate. The analysis of the timecourse samples by f.a.b.-m.s. indicated a rate-enhancement factor of approx. 4-fold, with the disappearance of native insulin disulphide-bridge signals in less than $1 \mathrm{~h}$ and the formation of precipitate, defined here as bridged B-chain signals, within $15 \mathrm{~min}$.

\section{DISCUSSION}

A series of experiments were carried out on the reduction and scrambling of the disulphide bridges of insulin, to examine the capability of a new method of disulphide-bridge assignment for the study of protein refolding. The method is based on high-mass

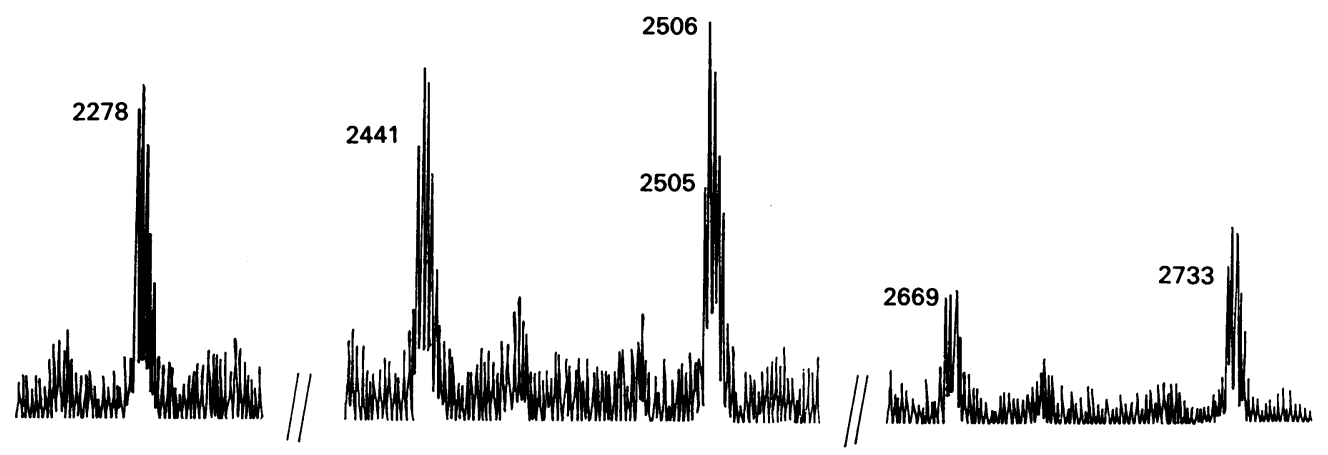

Fig. 3. Partial f.a.b.-m.s. map showing the high-mass region of a peptic digest of the precipitate from an insulin-scrambling experiment taken at the $2 \mathrm{~h}$ incubation point

The spectrum clearly shows only the presence of signals derived from disulphide bridges between B-chains, namely Cys-B7-Cys-B7 and Cys-B7-Cys-B19. For interpretation see the text and Table 2. 
m.s. for the characterization of disulphide-bridged peptides according to their precise molecular masses.

The success of this new approach is clearly demonstrated here, since we are able to monitor the extent of reduction, the disappearance of signals representing the normal 'native' disulphide bridges and the appearance of signals diagnostic for the scrambled state.

Furthermore, our procedures have led to the characterization of the precipitate, the formation of which during the scrambling of insulin was first reported by Anfinsen's group: Givol et al. [10] demonstrated that incubation of insulin with PDI produced a precipitate, which these authors interpreted as being due to scrambling of the native disulphides, although no definitive structural characterization of the precipitate was made. The m.s. analysis reported in the present paper unambiguously allowed the structural definition of this precipitate as deriving from disulphide-bridged B-chains, corresponding to Cys-B7-Cys-B7 and Cys-B7-Cys-B19. Further characterization of the precipitate by SDS/PAGE showed the presence of a band with a molecular mass corresponding to a B-chain dimer (results not shown). The occurrence of such a dimer during A-chain/B-chain reconstitution procedures in the preparation of recombinant human insulin has recently been independently reported [11].

On the basis of the m.s. data, the formation of the two B-chain dimers observed, containing Cys-B7-Cys-B7 and Cys-B7-CysB19 interchain bonds, appears to be a consequence of $(a)$ the substoichiometric amount of DTT leading to a complex redox mixture containing partially reduced insulin moieties together with reduced and oxidized DTT, $(b)$ the B7 cysteine thiol anion apparently being the preferred nucleophile in solution and $(c)$ the insolubility of the B-chain dimers, once formed, removing them from the complex redox reaction mixture, thus driving the products to B-chain dimers.

Experiments with PDI were carried out to examine the predicted catalysis of the processes observed and to ask the question whether any differences could be detected in the PDI-catalysed scrambled disulphide bonds. No differences were observed in the PDI and non-PDI spectra with respect to the disulphide-bridge signals, but similar spectra were obtained approximately 4 times more quickly in the time-course experiments, indicating a definite rate enhancement of the formation of new disulphide bonds.

This work shows that our procedures for assigning disulphide bridges in proteins can be applied to the study of protein reduction and re-oxidation, denaturation and refolding, by sampling the protein conformers on a time course and rapidly freezing the structural picture at any time by precipitation/ digestion and high-mass m.s. analysis. The procedure is rapid and the interpretation definitive, since a precise physicochemical quantity, the mass of a signal in the spectrum, is diagnostic for the presence of a particular disulphide linkage in the protein. The assignment of signals can of course be confirmed by techniques that we have described previously [8], such as reduction on the probe or steps of Edman degradation followed by m.s. analysis.

We are grateful for M.R.C. Programme Grant support, for a N.A.T.O. Travel Fellowship and for a grant from the Italian Ministry of Education. G. M. also acknowledges a FORMEZ (Rome) fellowship.

\section{REFERENCES}

1. Creighton, T. E. (1985) J. Phys. Chem. 89, 2452-2459

2. King, J. (1986) Bio/Technology 4, 297-303

3. Creighton, T. E. (1978) Prog. Biophys. Mol. Biol. 33, 231-297

4. Creighton, T. E. \& Goldenberg, D. P. (1984) J. Mol. Biol. 179, 527-545

5. Creighton, T. E. (1979) J. Mol. Biol. 129, 411-431

6. Marks, C. B., Naderi, H., Kosen, P. A., Kuntz, I. D. \& Anderson, D. (1987) Science 235, 1370-1373

7. Sheraga, H. A., Konishi, Y., Rothwarf, D. M. \& Mui, P. W. (1987) Proc. Natl. Acad. Sci. U.S.A. 84, 5740-5744

8. Morris, H. R. \& Pucci, P. (1985) Biochem. Biophys. Res. Commun. 126, $1122-1128$

9. Blundell, T., Dodson, G., Hodgkin, D. \& Mercola, D. (1972) Adv. Protein Chem. 26, 279-296

10. Givol, D., De Lorenzo, F., Goldberger, R. F. \& Anfinsen, C. B. (1965) Proc. Natl. Acad. Sci. U.S.A. 53, 676-684

11. Toren, P., Smith, D., Chance, R. \& Hoffman, J. (1988) Anal. Biochem. 169, 287-299 